\title{
Voxel-based morphometry in patients with mood disorder bipolar I mania in comparison to normal controls
}

\author{
Hosam Abozaid Yousef ${ }^{*}$ (D), Yasser Mohamed Bader-Eldein ElSerogy ${ }^{2}$, Sherif Mohamed Abdelal ${ }^{1}$ and \\ Shaza Ragab Abdel-Rahman'
}

\begin{abstract}
Background: Neuroimaging is used to study brain structural alterations in neuropsychiatric diseases including bipolar disorder (BD). Voxel-based morphometry (VBM) quantifies structural changes detected in magnetic resonance imaging (MRI). The aim of this study was to identify brain structural changes in patients with mood disorder bipolar I mania, compared to healthy controls; and detect any correlations between volumetric findings and different clinical aspects of the disease. VBM was used to identify structural changes in 24 patients with bipolar I mania compared to 16 controls. Young Mania Rating Scale (YMRS) was used to evaluate clinical severity of BD. $t$ test was used to compare differences in volumetric data and Spearman's rank correlation coefficient was used to detect potential correlations between volumetric data and clinical parameters of BD.
\end{abstract}

Results: Compared to controls, BD patients had significantly larger right globus pallidus and right lateral ventricle. There was significant correlation between volumetric data of different brain structures and clinical criteria of BD including age of onset, illness duration, YMRS, number of manic attacks, and duration of the last attack.

Conclusions: VBM could address specific structural findings in bipolar I mania that may contribute to pathophysiology of the disease and show significant correlation with different clinical aspects of the disease.

Trial registration: This clinical trial was registered at ClinicalTrials.gov under registration number NCT03181698, registered 11 June 2017.

Keywords: Bipolar I mania, Voxel-based morphometry, MRI, Volumetry

\section{Background}

Bipolar disorder (BD) is a common psychiatric illness characterized by disruptive mood swings presented as episodes of mania/hypomania with or without depressive episodes, with normal mood in-between episodes [1]. It is characterized by affective instability and cognitive deficits [2].

The exact pathophysiology of this mental illness is still not fully understood. It has been proved that lesions in some specific neuroanatomical areas of the brain, e.g., stroke or brain tumors, may lead to the development of secondary mental illness. With great development in structural

\footnotetext{
* Correspondence: hosameldeen68@aun.edu.eg

${ }^{1}$ Radiodiagnosis Department, Faculty of Medicine, Assiut University Hospitals,

Assiut University, Assiut University Street, Assiut 71515, Egypt

Full list of author information is available at the end of the article
}

neuroimaging, recent neurological studies are increasingly concerned with studying the relationship between the anatomy and pathophysiology of mental diseases [3].

The most widely used clinical criteria for diagnosis of mania is from the American Psychiatric Association's Diagnostic and Statistical Manual of mental disorders (DSM-IV), and the most commonly used criteria for grading of the severity of manic episode is the Young Mania Rating Scale (YMRS) [4].

Recent advances in multimodal neuroimaging contributed much to the understanding of the relation between structural brain abnormalities and behavioral disturbances [5]. Neuroimaging studies in bipolar disorder have documented structural and functional alterations in the brain, including gray matter morphology, white matter integrity, and local/global neuronal connectivity [6]. 
Brain morphometry is the study of size and shape of the brain and its structures, based on non-invasive imaging data obtained from magnetic resonance imaging (MRI). Voxel-based morphometry (VBM) $[7,8]$ is an unbiased automated computer-based technique that enables voxel-wise measurement of brain structures. The concept of VBM is based on three basic steps which include tissue classification, spatial normalization, and spatial smoothing, and the final step in a VBM analysis is to perform voxel-wise statistical tests.

\section{Aim of the work}

The aim of this study was to determine structural anatomical changes that could be associated with mood disorder bipolar I mania through detection of volumetric changes in different parts of the brain using a fully automated voxel-based morphometric analysis. The study also tried to detect any correlation between volumetric findings in patients with $\mathrm{BD}$ and different clinical aspects of the disease.

\section{Methods}

\section{Ethics statement}

The protocol, design, and procedures of this prospective study were approved by the institutional Ethical Committee Board. Informed written consents were obtained from the parents or the responsible patients' legal guardians. Informed written consents were also obtained from the healthy control subjects who were included in the study. Confidentiality was maintained throughout the whole study.

\section{Subjects}

Patients

Twenty-four patients diagnosed with mood disorder bipolar "I" manic episodes were recruited. The patients were assessed by structured clinical interview for DSMIV criteria. The severity of the manic episode was assessed using YMRS.

\section{Controls}

Carefully selected 16 healthy subjects matched for age, gender, socioeconomic status, and education were included as controls in the study. None of these subjects had neurological illness, and they were provisionally evaluated by experienced psychiatrists to exclude any psychiatric or neurologic disorder.

\section{Methods}

\section{MRI \& VBM evaluation}

All MRI examinations were performed on a 1.5 Tesla MRI scanner (Achieva, Philips Medical Systems, Best, The Netherlands), using a 16-channel phased-array head coil. Following the multiplanar localizer sequence, the
MR scanning protocol included routinely: axial FLAIR, axial diffusion-weighted, and axial 3D-T1W-FFE. The FLAIR and diffusion-weighted images were carefully inspected to exclude any gross anatomical abnormality or pathological changes in the brain. The highresolution l-mm-thick 3D-T1W-FFE images were used for the voxel-based morphometry study. Demonstrative 1-mm 3D-TFE images of a patient and a control subject are shown in Figs. 1a and 2a, respectively.

For morphometric evaluation, the 3D-T1W-FFE images were converted from the standard digital imaging and communication (DICOM) format $(. \mathrm{dcm})$ to the Neuroimaging Informatics Technology Initiative (NIfTI) format (.nii) suitable for analysis and post-processing. We used MRIcron software (Chris Rodren, 2014, Neuropsychology Lab, University of South Carolina, USA). MRIcron is a cross-platform NIfTI format image viewer which provides "dcm2nii tool" for converting DICOM images to NIfTI format. During the step of format conversion, the output nii data were chosen to be generated in a compressed form.

In all the processes, DICOM data were anonymized to protect the privacy of the participants. The anonymization process was also performed using MRIcron.

The compressed nii data were then uploaded through the internet to the online-based website of volBrain (https://volbrain.upv.es/) for analysis. volBrain is a fully automated segmentation pipeline developed by Manjón (Universidad Politécnica de Valencia, Spain) and Coupé (Université de Bordeaux, France) [9].

The technique of data processing, as provided by the pipeline developers, consists of a cascade of steps that include image de-noising, field and/or radiofrequency inhomogeneity correction, multiple-atlas label registration, intracranial cavity extraction, tissue classification, hemispheric segmentation, and finally, subcortical structures segmentation.

After completion of the analysis process, an electronic report was generated for each uploaded case containing the volumes of the main intracranial structures including the cerebrospinal fluid (CSF), cerebral gray matter (GM), and white matter (WM), as well as volume information of macroscopic areas such as the cerebral hemispheres, the cerebellum, and brainstem. The report also included the calculated volumes of different subcortical structures. Labeled colored graphical maps of the abovementioned anatomical structures were also provided for each case.

\section{Statistical analysis}

We used $t$ test to quantify the differences in the volumetric data of different parts of the brain between BD patients and the controls. We also used Spearman's rank correlation coefficient within the $\mathrm{BD}$ patients' 


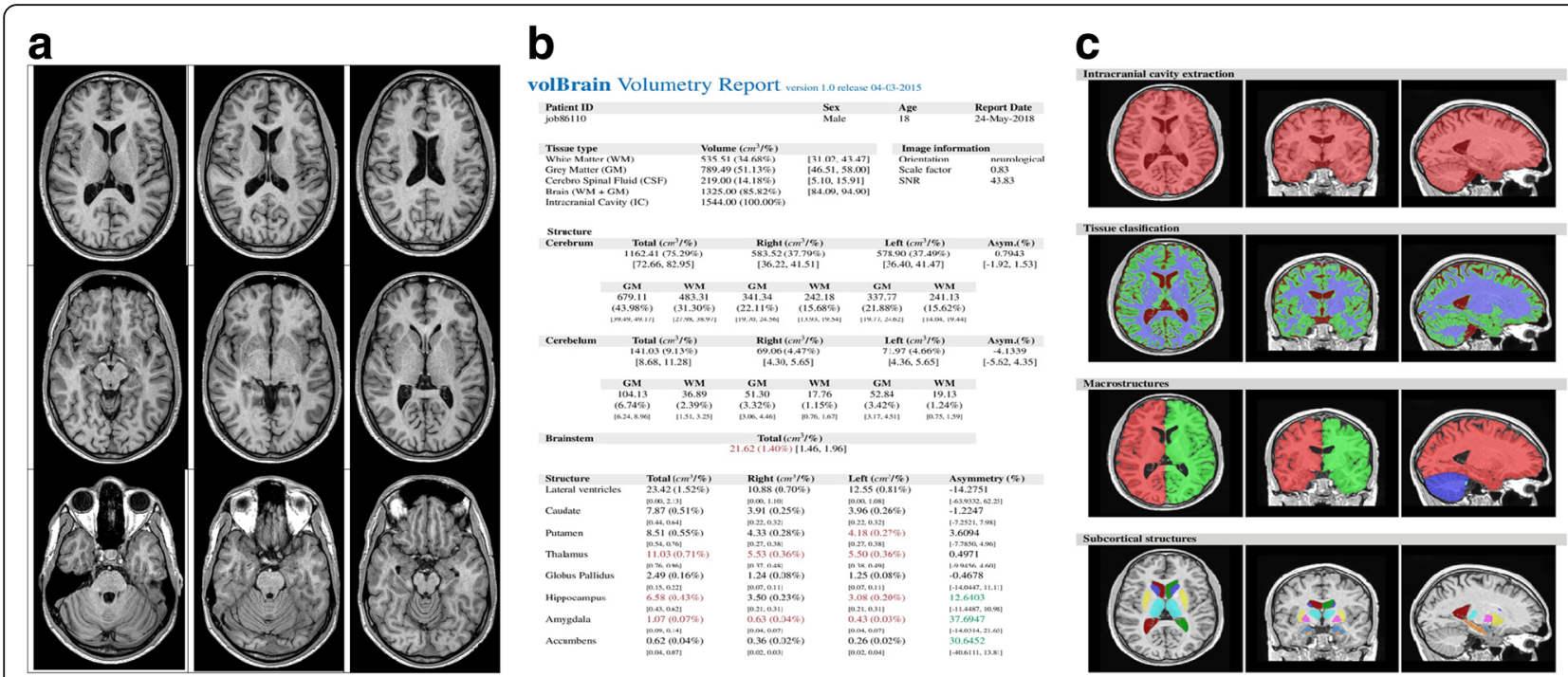

Fig. 1 a High-resolution 1-mm 3D-TFE images of an 18-year-old male patient, used for the voxel-based morphometric evaluation. $\mathbf{b}$ Volbrain electronically generated volumetric data in an 18-year-old male patient. c Volbrain-generated colored graphical maps of the different intracranial structures of an 18-year-old male patient

group for detection of potential correlations between the volumetric data and the clinical parameters including the patient's age, age of onset of $\mathrm{BD}$, duration of illness, number of manic attacks, duration of the last attack, and YMRS as covariates.

\section{Results}

\section{Demographic data}

The study population included two groups of subjects; 24 patients with well-characterized bipolar disorder type I, and 16 healthy control subjects. In the patients' group, there were 23 male patients and 1 female patient; their ages ranged from 18 to 47 years (Mean $\pm \mathrm{SD}=28.00 \pm$ 9.09). The control group included 15 male subjects and 1 female; their age range was $19-46$ years (Mean $\pm \mathrm{SD}=$ $31.56 \pm 7.30$; Table 1).

\section{Clinical data}

The age of onset of $\mathrm{BD}$ in the patients ranged from 12 to 46 years, with mean \pm SD of $23.33 \pm 8.34$. The duration of illness ranged from 1 to 16 years, with mean \pm $\mathrm{SD}$ of $4.87 \pm 4.34$. The number of manic episodes in
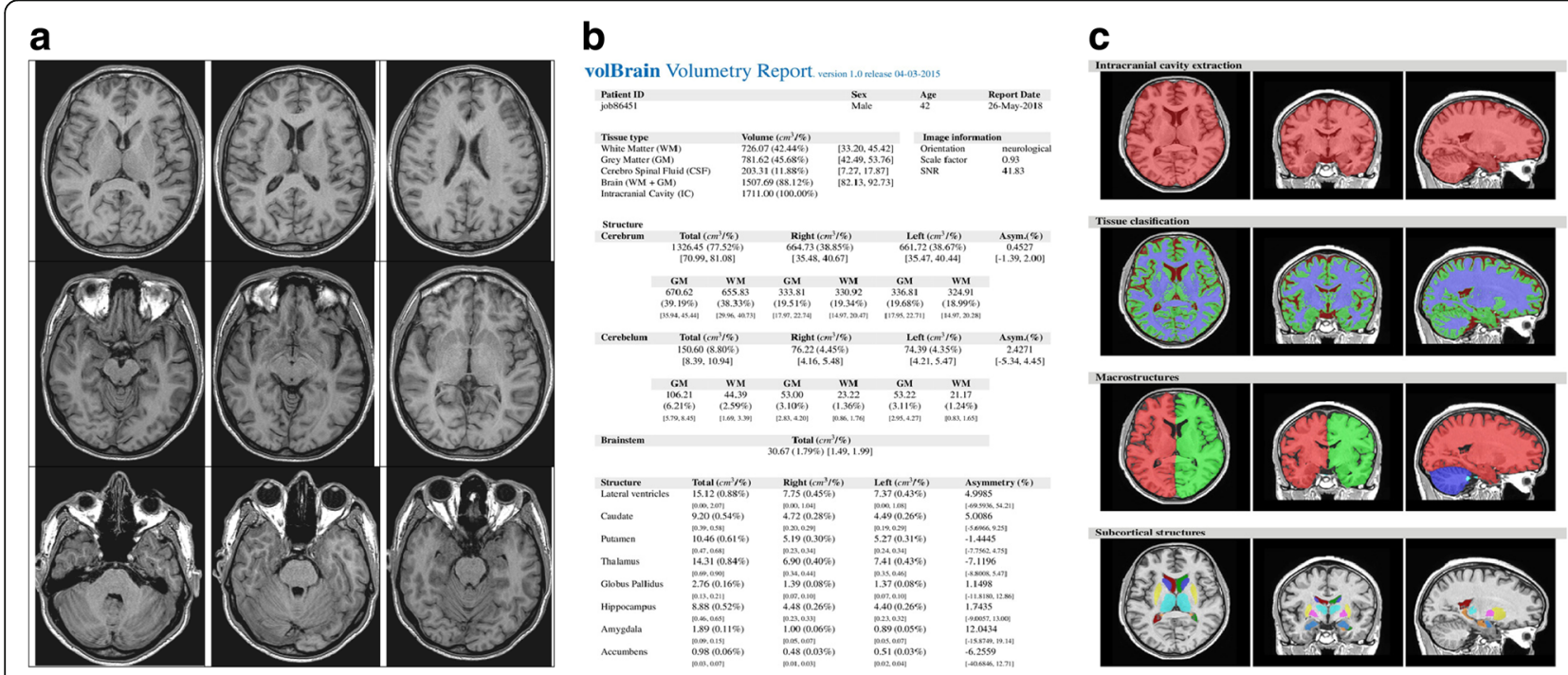

Fig. 2 a High-resolution 1-mm 3D-TFE images of a 42-year-old male control. b Volbrain electronically generated volumetric data in a 42-year-old male control. c Volbrain-generated colored graphical maps of the different intracranial structures of a 42-year-old male control 
Table 1 Demographic data of patients and healthy controls

\begin{tabular}{|c|c|c|c|c|c|}
\hline \multirow[t]{2}{*}{ Personal data } & \multicolumn{2}{|c|}{$\begin{array}{l}\text { Patients } \\
(\text { No. }=24)\end{array}$} & \multicolumn{2}{|c|}{$\begin{array}{l}\text { Controls } \\
(\text { No. }=16)\end{array}$} & \\
\hline & No. & Percentage (\%) & No. & Percentage (\%) & \\
\hline \multicolumn{6}{|l|}{ Gender } \\
\hline Male & 23 & 95.8 & 14 & 87.5 & 0.615 \\
\hline Female & 1 & 4.2 & 2 & 12.5 & \\
\hline \multicolumn{6}{|l|}{ Age (years) } \\
\hline Mean \pm SD & \multicolumn{2}{|c|}{$28.00 \pm 9.09$} & \multicolumn{2}{|c|}{$31.56 \pm 7.30$} & 0.118 \\
\hline Range & \multicolumn{2}{|c|}{$18.0-47.0$} & \multicolumn{2}{|c|}{$19.0-46.0$} & \\
\hline \multicolumn{6}{|l|}{ Marital status } \\
\hline Single & 13 & 54.2 & 8 & 50.0 & 0.655 \\
\hline Married & 10 & 41.7 & 8 & 50.0 & \\
\hline Divorced & 1 & 4.2 & 0 & 0.0 & \\
\hline \multicolumn{6}{|c|}{ Level of education } \\
\hline Illiterate & 3 & 12.5 & 0 & 0.0 & $0.013^{*}$ \\
\hline Primary & 7 & 29.2 & 1 & 6.3 & \\
\hline Preparatory & 5 & 20.8 & 1 & 6.3 & \\
\hline Secondary & 7 & 29.2 & 6 & 37.5 & \\
\hline University & 2 & 8.3 & 8 & 50.0 & \\
\hline \multicolumn{6}{|l|}{ Handedness } \\
\hline Right & 19 & 79.2 & 13 & 81.3 & 0.872 \\
\hline Left & 5 & 20.8 & 3 & 18.8 & \\
\hline
\end{tabular}

* Significant

patients ranged from 1 to 7 with mean \pm SD of $3.08 \pm$ 1.53. The duration of the last episode ranged from 2 to $22 \mathrm{~h}$, with mean \pm SD of $7.29 \pm 5.41$. The Young Mania Rating Scale of patients ranged from 17 to 45 with mean \pm SD of $32.04 \pm 6.97$ (Table 2). Among the 24 patients of the study, 9 had positive history of substance abuse.

\section{Volumetric MRI data}

Volumetric differences between BD patients and healthy controls are presented in Table 3. The mean volume \pm SD of the right globus pallidus was found significantly larger in the patients group than in the healthy controls $(0.99 \pm 0.19$ and $0.84 \pm 0.30$, respectively; $p=0.03$; Fig. 3 ). Although the mean volume \pm $\mathrm{SD}$ of the left globus pallidus was found relatively larger in the patients' group compared to the healthy controls, the difference was not significant $(0.99 \pm 0.20$ and $0.84 \pm 0.29$, respectively; $p=0.07$ ).

The calculated mean volumes of other subcortical structures, namely the caudate nucleus, putamen, thalamus, amygdale, and hippocampus were all relatively larger in the patients group than in the healthy controls, but the differences were statistically insignificant as follows:
Table 2 Clinical data of patients of the study

\begin{tabular}{lll}
\hline & No. $(n=24)$ & Percentage (\%) \\
\hline Drug dependence & & $37.5 \%$ \\
Yes & 9 & $62.5 \%$ \\
No & 15 & $33.3 \%$ \\
Type of drugs & & $11.1 \%$ \\
Hashish & 3 & $55.6 \%$ \\
Tramadol & 1 & \\
Both & 5 & \\
No. of manic episodes & \\
Mean \pm SD & $3.08 \pm 1.53$ & \\
Range & $1.0-7.0$ (times) \\
Duration of illness (years) & \\
Mean \pm SD & $4.87 \pm 4.34$ & \\
Range & $1.0-16.0$ (years) \\
Age of onset (years) & \\
Mean \pm SD & $23.33 \pm 8.34$ & \\
Range & $12.0-46.0$ (years) \\
Young Mania Rating Scale (YMRS) \\
Mean \pm SD & $37.04 \pm 6.97$ & \\
Range & $23.0-45.0$ & \\
\hline
\end{tabular}

The mean volume \pm SD of both right and left caudate nuclei in the patients were $3.55 \pm 0.46$ and $3.58 \pm 0.42$, respectively, and in the healthy controls were $3.52 \pm 0.52$ and $3.44 \pm 0.53$, respectively; $p=0.83$ for the right caudate and 0.31 for the left caudate.

The mean volume \pm SD of both right and left putamen in the patients were $4.07 \pm 0.36$ and $4.00 \pm 0.35$, respectively, and in the healthy controls were $3.93 \pm 0.80$ and $3.94 \pm 0.75$, respectively; $p=0.33$ for the right putamen and 0.49 for the left putamen.

The mean volume \pm SD of both right and left thalami in the patients were $5.34 \pm 0.62$ and $5.46 \pm 0.69$, respectively, and in the healthy controls were $3.61 \pm 0.42$ and $3.54 \pm 0.44$, respectively; $p=0.51$ for the right thalamus and 0.66 for the left thalamus.

The mean volume \pm SD of the right and left hippocampus in the patients were $3.62 \pm 0.36$ and $3.72 \pm 0.41$, respectively, and in the healthy controls were $5.26 \pm 0.70$ and $5.42 \pm 0.71$, respectively; $p=0.85$ for the right hippocampus and 0.21 for the left hippocampus.

The mean volume \pm SD of both right and left amygdala in the patients were $0.57 \pm 0.16$ and $0.58 \pm 0.15$, respectively, and in the healthy controls were $0.55 \pm 0.17$ and $0.54 \pm 0.18$, respectively; $p=0.31$ for the right hippocampus and 0.39 for the left hippocampus.

There was no statistically significant difference in the mean volume $\pm \mathrm{SD}$ of the right and left cerebral 
Table 3 Volumetric data of different brain structures in patients and controls

\begin{tabular}{|c|c|c|c|}
\hline & $\begin{array}{l}\text { Patient } \\
\text { group } \\
(\text { No. }=24)\end{array}$ & $\begin{array}{l}\text { Control } \\
\text { group } \\
(\text { No. }=16)\end{array}$ & $p$ value \\
\hline Rt caudate & $3.6 \pm 0.5$ & $3.5 \pm 0.5$ & 0.9 \\
\hline Lt caudate & $3.6 \pm 0.4$ & $3.4 \pm 0.5$ & 0.3 \\
\hline Rt putamen & $4.1 \pm 0.4$ & $3.9 \pm 0.8$ & 0.5 \\
\hline Lt putamen & $4 \pm 0.3$ & $3.9 \pm 0.8$ & 0.7 \\
\hline Rt thalamus & $5.3 \pm 0.6$ & $5.2 \pm 0.7$ & 0.8 \\
\hline Lt thalamus & $5.5 \pm 0.7$ & $5.4 \pm 0.7$ & 0.9 \\
\hline Rt globus pallidus & $1 \pm 0.2$ & $0.8 \pm 0.3$ & $0.05^{*}$ \\
\hline Lt globus pallidus & $1 \pm 0.2$ & $0.8 \pm 0.3$ & 0.06 \\
\hline Rt hippocampus & $3.6 \pm 0.3$ & $3.6 \pm 0.4$ & 0.9 \\
\hline Lt hippocampus & $3.7 \pm 0.4$ & $3.5 \pm 0.4$ & 0.2 \\
\hline Rt amygdala & $0.5 \pm 0.1$ & $0.6 \pm 0.2$ & 0.8 \\
\hline Lt amygdala & $0.6 \pm 0.1$ & $0.5 \pm 0.2$ & 0.4 \\
\hline Rt cerebral hemisphere & $530 \pm 61$ & $524 \pm 76$ & 0.8 \\
\hline Lt cerebral hemisphere & $534 \pm 62$ & $525 \pm 74$ & 0.7 \\
\hline Rt cerebral gray matter & $291 \pm 56$ & $288 \pm 44$ & 0.8 \\
\hline Lt cerebral gray matter & $389 \pm 56$ & $292 \pm 45$ & 0.8 \\
\hline Rt cerebral white matter & $240 \pm 69$ & $237 \pm 41$ & 0.8 \\
\hline Lt cerebral white matter & $240 \pm 74$ & $233 \pm 40$ & 0.7 \\
\hline Rt lateral ventricle & $8.76 \pm 4.88$ & $5.97 \pm 4.63$ & $0.03^{*}$ \\
\hline Lt lateral ventricle & $7.43 \pm 3.34$ & $6.44 \pm 4.29$ & 0.18 \\
\hline Rt cerebellar hemisphere & $65 \pm 13$ & $65 \pm 9$ & 0.9 \\
\hline Lt cerebellar hemisphere & $67 \pm 12$ & $65 \pm 9$ & 0.7 \\
\hline
\end{tabular}

$R t$ right, $L t$ left

Both values in boldface are significant, equal to or below 0.05 * Significant hemispheres between patients $(530.52 \pm 60.92$ and $533.88 \pm 62.54$, respectively) and healthy controls ( $524.75 \pm 75.87$ and $525.42 \pm 74.19$, respectively); $p=0.73$ for the right cerebral hemisphere and $p=0.58$ for the left cerebral hemisphere.

Also, no significant difference was detected in the mean volume $\pm S D$ of the cortical gray matter of both right and left cerebral hemispheres between patients $(290.79 \pm 56.30$ and $293.73 \pm 58.00$, respectively) and healthy controls $(287.77 \pm 43.98$ and $291.66 \pm 44.90$, respectively); $p=0.44$ for the right cerebral hemisphere and $p=0.45$ for the left cerebral hemisphere.

There was no significant difference in the mean volume \pm SD of the white matter of both right and left cerebral hemispheres between patients $(239.72 \pm 68.62$ and $240.15 \pm 74.27$, respectively) and healthy controls (236.98 \pm 41.55 and $233.76 \pm 39.99$, respectively); $p=0.60$ for the right cerebral hemisphere and $p=0.82$ for the left cerebral hemisphere.

The mean volume \pm SD of the right lateral ventricle was significantly larger than in the healthy controls $(8.76 \pm 4.88$ and $5.97 \pm 4.63$, respectively; $p=0.03$; Fig. 4); however, although the mean volume \pm SD of the left lateral ventricle was relatively larger in the patients' group compared to healthy controls, the difference was not statistically significant $(7.43 \pm 3.34$ and $6.44 \pm 4.29$, respectively; $p=0.18$ ).

Regarding the cerebellum, there was no significant difference in the mean volume \pm SD of the right and left cerebellar hemispheres between patients $(65.25 \pm 13.25$ and $67.10 \pm 12.17$, respectively) and healthy controls $(65.25 \pm 8.81$ and $65.63 \pm 8.66$, respectively); $p=0.76$ for the right cerebellar hemisphere and $p=0.54$ for the left cerebellar hemisphere.

Examples of the electronically generated reports and colored graphical maps of the volumetric data for a patient and a control subject are demonstrated in Figs. 1b and $\mathrm{c}$ and $2 \mathrm{~b}$ and $\mathrm{c}$, respectively.

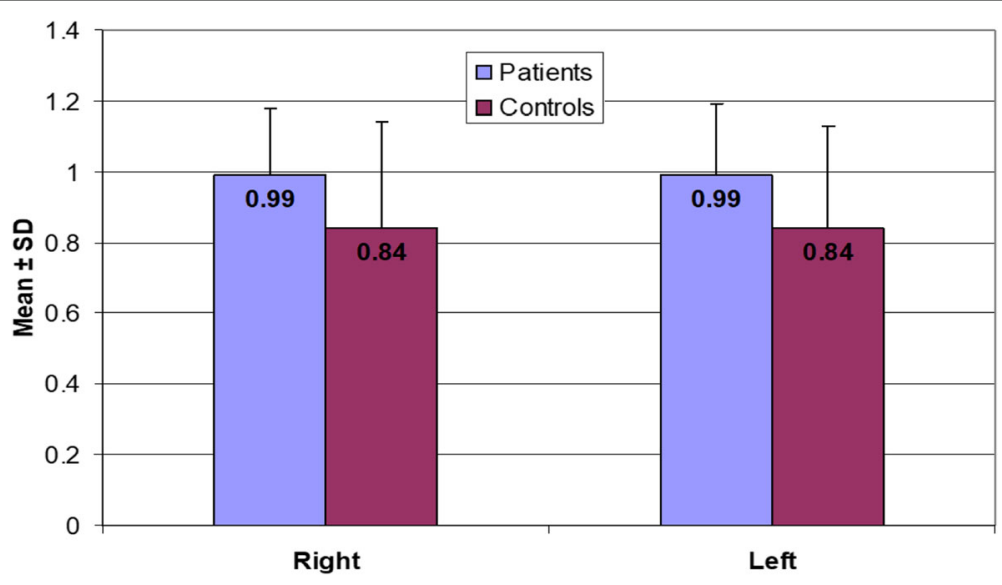

Fig. 3 Volumetric data of the globus pallidus in healthy patients and controls 


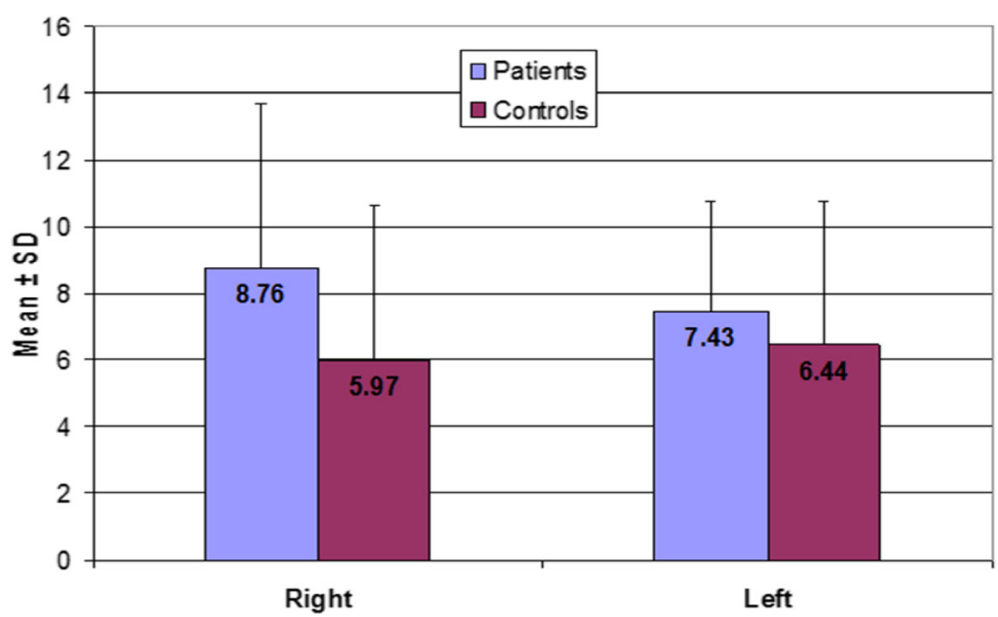

Fig. 4 Volumetric differences in the lateral ventricles between patients and controls

\section{The correlation between volumetric data and some} clinical criteria in BD patients

The Spearman's analysis of the correlation between the mean volumes of different anatomical structures in the brain yielded the following results (Table 4):

There was a significant negative correlation between the mean volume of the right caudate nucleus and the patient's age $(r=-0.42, p=0.04)$, as well as the age of onset of $\mathrm{BD}(r=-0.44, p=0.03)$.

There was a highly significant negative correlation between the mean volume of the right putamen and the patient's age $(r=-0.62, p=0.001)$ and significant negative correlation between the mean volume of the right putamen and the age of onset of $\mathrm{BD}(r=-0.46, p=$ $0.02)$. Similar findings were found in the left putamen where there is highly significant negative correlation between the mean volume of the left putamen and the patient's age $(r=-0.60, p=0.002)$ and significant negative correlation between the mean volume of the left putamen and the age of onset of $\mathrm{BD}(r=-0.43, p=0.02)$.

Regarding the globus pallidus, there was a highly significant negative correlation between the mean volume of the right globus pallidus and the patient's age ( $r=-0.55, p=0.005)$. The mean volume of the left globus pallidus shows a significant negative correlation with the patient's age $(r=-0.43, p=0.03)$, Also, it shows significant negative correlation with the age of onset of BD $(r=-0.46, p=0.02)$.

There is a significant negative correlation between the mean volume of the right thalamus and YMRS of the patient $(r=-0.40, p=0.04)$. The mean volume of the left thalamus shows highly significant negative correlation with the YMRS of the patient $(r=-0.58$, $p=0.003)$.
The mean volume of the left hippocampus shows significant positive correlation with the YMRS of the patient $(r=0.46, p=0.02)$.

The mean volume of the right amygdala shows significant negative correlation with the number of manic attacks $(r=-0.47, p=0.02)$.

The mean total volume of each cerebral hemisphere shows significant negative correlation with the patient's age as well as the YMRS of the patient. For the right cerebral hemisphere, the values were $r=-0.43, p=0.03$ and $r=-0.47, p=0.02$, respectively; and for the left cerebral hemisphere, the values were $r=-0.45, p=0.02$ and $r=-0.48, p=0.01$, respectively.

The mean volume of the gray matter of each cerebral hemisphere shows highly significant negative correlation with the patient's age. For the right cerebral hemisphere, the values were $r=-0.63, p=0.001$ and for the left cerebral hemisphere, the values were $r=0.62, p=0.001$. Also, the volume of the gray matter of each cerebral hemisphere shows significant negative correlation with the number of attacks as well as the duration of illness. The values for the right cerebral hemisphere were $r=$ $-0.49, p=0.01$ and $r=-0.47, p=0.02$, respectively, and for the left cerebral hemisphere, were $r=-0.46, p=0.02$ and $r=-0.49, p=0.01$, respectively.

The mean volume of the white matter of each cerebral hemisphere shows highly significant negative correlation with the patient's YMRS. For the right cerebral hemisphere, the values were $r=-0.68, p=0.001$ and for the left cerebral hemisphere, the values were $r=0.65$, $p=0.001$. Also, the volume of the white matter of the left cerebral hemisphere shows significant negative correlation with the duration of the last attack of mania $(r=-0.42, p=0.04)$. 
Table 4 Correlation between clinical data and volumetric data of different brain structures

\begin{tabular}{|c|c|c|c|c|c|c|}
\hline & Age (years) & No. of attacks & Duration of illness & Age of onset (years) & $\begin{array}{l}\text { Duration of } \\
\text { last episode }\end{array}$ & $\begin{array}{l}\text { Young Mania } \\
\text { Rating scale }\end{array}$ \\
\hline \multicolumn{7}{|l|}{ Rt caudate } \\
\hline$r$ value & -0.420 & 0.051 & 0.108 & -0.441 & -0.058 & 0.373 \\
\hline$p$ value & $0.041^{*}$ & 0.812 & 0.615 & $0.031^{*}$ & 0.789 & 0.072 \\
\hline \multicolumn{7}{|l|}{ Lt caudate } \\
\hline$r$ value & -0.360 & 0.086 & 0.098 & -0.351 & -0.184 & 0.384 \\
\hline$p$ value & 0.084 & 0.690 & 0.649 & 0.093 & 0.391 & 0.064 \\
\hline \multicolumn{7}{|c|}{ Rt putamen } \\
\hline$r$ value & -0.621 & -0.220 & -0.223 & -0.465 & 0.331 & -0.004 \\
\hline$p$ value & $0.001^{* *}$ & 0.302 & 0.295 & $0.022^{*}$ & 0.114 & 0.986 \\
\hline \multicolumn{7}{|c|}{ Lt putamen } \\
\hline$r$ value & -0.606 & -0.195 & -0.262 & -0.416 & 0.260 & 0.090 \\
\hline$p$ value & $0.002^{* *}$ & 0.360 & 0.215 & $0.043^{*}$ & 0.221 & 0.677 \\
\hline \multicolumn{7}{|c|}{ Rt thalamus } \\
\hline$r$ value & -0.119 & -0.045 & 0.008 & -0.125 & -0.281 & 0.407 \\
\hline$p$ value & 0.578 & 0.833 & 0.972 & 0.560 & 0.184 & $0.049^{* *}$ \\
\hline \multicolumn{7}{|c|}{ Lt thalamus } \\
\hline$r$ value & -0.091 & -0.127 & -0.034 & -0.091 & -0.362 & 0.581 \\
\hline$p$ value & 0.672 & 0.555 & 0.876 & 0.671 & 0.082 & $0.003^{* *}$ \\
\hline \multicolumn{7}{|c|}{ Rt globus pallidus } \\
\hline$r$ value & -0.552 & -0.329 & -0.384 & -0.341 & 0.310 & -0.295 \\
\hline$p$ value & $0.005^{*}$ & 0.117 & 0.064 & 0.103 & 0.140 & 0.162 \\
\hline \multicolumn{7}{|c|}{ Lt globus pallidus } \\
\hline$r$ value & -0.437 & -0.141 & -0.166 & -0.460 & 0.159 & -0.064 \\
\hline$p$ value & $0.033^{*}$ & 0.512 & 0.439 & $0.024^{*}$ & 0.458 & 0.768 \\
\hline \multicolumn{7}{|c|}{ Rt hippocumpus } \\
\hline$r$ value & -0.155 & -0.229 & -0.309 & 0.123 & -0.062 & 0.307 \\
\hline$p$ value & 0.470 & 0.282 & 0.141 & 0.567 & 0.775 & 0.145 \\
\hline \multicolumn{7}{|c|}{ Lt hippocumpus } \\
\hline$r$ value & -0.186 & -0.029 & -0.167 & -0.083 & 0.060 & 0.465 \\
\hline$p$ value & 0.384 & 0.891 & 0.435 & 0.700 & 0.779 & $0.022^{*}$ \\
\hline \multicolumn{7}{|c|}{ Rt amygdala } \\
\hline$r$ value & -0.132 & -0.471 & -0.251 & 0.073 & 0.032 & -0.091 \\
\hline$p$ value & 0.540 & $0.020^{*}$ & 0.237 & 0.735 & 0.882 & 0.672 \\
\hline \multicolumn{7}{|c|}{ Lt amygdala } \\
\hline$r$ value & -0.152 & -0.192 & -0.145 & -0.046 & -0.254 & 0.139 \\
\hline$p$ value & 0.478 & 0.369 & 0.498 & 0.831 & 0.231 & 0.516 \\
\hline \multicolumn{7}{|c|}{ Rt cerebral hemisphere } \\
\hline$r$ value & -0.434 & -0.157 & -0.189 & -0.336 & -0.214 & 0.472 \\
\hline$p$ value & $0.034^{*}$ & 0.465 & 0.377 & 0.108 & 0.315 & $0.020^{*}$ \\
\hline \multicolumn{7}{|c|}{ Lt cerebral hemisphere } \\
\hline$r$ value & -0.451 & -0.130 & -0.218 & -0.330 & -0.190 & 0.484 \\
\hline$p$ value & $0.027^{*}$ & 0.545 & 0.307 & 0.115 & 0.373 & $0.016^{*}$ \\
\hline
\end{tabular}


Table 4 Correlation between clinical data and volumetric data of different brain structures (Continued)

\begin{tabular}{|c|c|c|c|c|c|c|}
\hline & Age (years) & No. of attacks & Duration of illness & Age of onset (years) & $\begin{array}{l}\text { Duration of } \\
\text { last episode }\end{array}$ & $\begin{array}{l}\text { Young Mania } \\
\text { Rating scale }\end{array}$ \\
\hline \multicolumn{7}{|c|}{ Rt lateral ventricle } \\
\hline$r$ value & -0.388 & -0.250 & -0.487 & $0.007^{*}$ & 0.101 & -0.198 \\
\hline$p$ value & 0.061 & 0.239 & $0.016^{*}$ & 0.973 & 0.640 & 0.353 \\
\hline \multicolumn{7}{|c|}{ Lt lateral ventricle } \\
\hline$r$ value & -0.378 & -0.207 & -0.384 & -0.044 & 0.037 & -0.283 \\
\hline$p$ value & 0.069 & 0.331 & 0.064 & 0.839 & 0.864 & 0.180 \\
\hline \multicolumn{7}{|c|}{ Rt cerebral gray matter } \\
\hline$r$ value & -0.633 & -0.498 & -0.470 & -0.269 & 0.017 & 0.080 \\
\hline$p$ value & $0.001^{* *}$ & $0.013^{*}$ & $0.021^{*}$ & 0.204 & 0.938 & 0.711 \\
\hline \multicolumn{7}{|c|}{ Lt cerebral gray matter } \\
\hline$r$ value & -0.626 & -0.462 & -0.492 & -0.238 & -0.006 & 0.107 \\
\hline$p$ value & $0.001^{* *}$ & $0.023^{*}$ & $0.015^{*}$ & 0.263 & 0.979 & 0.620 \\
\hline \multicolumn{7}{|c|}{ Rt cerebral white matter } \\
\hline$r$ value & 0.017 & 0.144 & 0.096 & -0.006 & -0.404 & 0.685 \\
\hline$p$ value & 0.936 & 0.501 & 0.656 & 0.979 & 0.050 & $0.000^{*}$ \\
\hline \multicolumn{7}{|c|}{ Lt cerebral white matter } \\
\hline$r$ value & -0.019 & 0.111 & 0.022 & -0.015 & -0.422 & 0.651 \\
\hline$p$ value & 0.929 & 0.605 & 0.920 & 0.943 & $0.040^{*}$ & $0.001^{*}$ \\
\hline \multicolumn{7}{|c|}{ Rt cerebellar hemisphere } \\
\hline$r$ value & -0.174 & -0.256 & -0.175 & 0.065 & -0.313 & 0.091 \\
\hline$p$ value & 0.417 & 0.227 & 0.412 & 0.762 & 0.137 & 0.674 \\
\hline \multicolumn{7}{|c|}{ Lt cerebellar hemisphere } \\
\hline$r$ value & -0.262 & -0.275 & -0.269 & 0.046 & -0.236 & 0.151 \\
\hline$p$ value & 0.216 & 0.193 & 0.204 & 0.830 & 0.266 & 0.482 \\
\hline
\end{tabular}

$R t$ right, $L t$ left

${ }^{*}$ Significant; ${ }^{* *}$ highly significant

The mean volume of the right lateral ventricle shows significant negative correlation with the duration of illness $(r=-0.48, p=0.01)$.

\section{Discussion}

Bipolar disorder is a psychiatric illness characterized by severe mood-state transitions from euthymic to depressed and manic states. The underlying pathophysiology of the disease represents a major research target for recent neuroimaging studies. There is a growing body of evidence that $\mathrm{BD}$ arises from abnormalities within brain systems that modulate emotional behavior implicating the frontal and limbic regions of the brain, which are central to emotional processing $[10,11]$.

Although the specific control of emotional function in humans is not completely understood, two networks appear to modulate emotional behavior. Both these networks map to specific striatal, pallidal, and thalamic brain areas to form feedback loops that process information and modulate the amygdala and other limbic areas. These two networks are likely central in the functional neuroanatomy of bipolar disorder [12-16]. Moreover, there is accumulated evidence suggesting that cortical-subcortical imbalance could represent a cause of bipolar disorder [17].

The prefrontal cortical areas that modulate human emotional function seem to do so within relatively independent prefrontal-striatal-pallidal-thalamic iterative circuits, the so-called cortico-striato-pallido-thalamocortical (CSPTC) circuits, which project back to the frontal lobe [18]. The globus pallidus is thought to be positioned at the heart of this CSPTC circuitry $[19,20]$.

Structural and functional neuroimaging studies on BD assume that abnormal prefrontal-subcortical-limbic connectivity cause cortical-limbic dysregulation reducing the inhibition of the ventral-limbic network and enhancing emotional responses [20-22]. Subcortical gray matter regions have been implicated in mood disorders, including BD [23]. Volumetric analysis of structural imaging data of the brain has been a mainstay investigation in psychiatry recently [24]. 
This study investigated structural neuroanatomical changes in patients with $\mathrm{BD}$ in comparison to healthy controls using VBM. VBM is continually being adapted as a research tool as it has advantages over conventional brain volume measurement in that it can detect small changes as it examines across the whole brain [25].

The primary focus was on the volumetric differences of different brain structures. Seven subcortical structures were explored: the caudate, putamen, globus pallidus, hippocampus, thalamus, and amygdala. The differences in the volumes of both cerebral and cerebellar hemispheres, the gray and white matter of both cerebral hemispheres, as well as the volume of the lateral ventricles were also assessed.

The findings of our study show that patients with BD had significantly larger mean volume of the right globus pallidus $(p=0.05)$ compared to healthy controls. BD patients also had significantly larger right lateral ventricles $(p=0.05)$ than healthy controls. None of the other evaluated subcortical structures showed significant volumetric differences between BD patients and control subjects. Also, no significant difference was detected between patients and controls concerning the mean total volume of both cerebral hemispheres, the mean gray matter and white matter volumes of both cerebral hemispheres, or the mean volume of both cerebellar hemispheres.

The findings of this study converge with multiple previous meta-analytical studies [26-28] which have shown enlarged lateral ventricles and globus pallidus in BD patients compared to healthy controls. The findings of this study also agree with previous research that described larger mean volume of the globus pallidus in BD patients compared to healthy controls $[29,30]$.

Concerning the volume of the lateral ventricle, the findings of this study are also concordant with those of a mega-analysis performed by Hallahan et al. [31] who reported enlargement of the right lateral ventricle volume in $\mathrm{BD}$ patients compared to healthy controls. Lateral ventricular enlargement was detected both in adolescent and adult BD patients, especially those with repeated manic episodes. This could suggest an underlying developmental mechanism, or might reflect the presence of a degenerative mechanism or progressive periventricular volume losses resulting from mood episodes [32, 33].

In $\mathrm{BD}$ patients, relative right hemisphere dysfunction has been postulated supported by reports of relatively greater impairment in visuospatial functioning and lateralization abnormalities [34]. The definite role of the globus pallidus in emotion and cognition is not clearly understood yet. Hence, potential explanations for the volume differences observed in the current study and similar research are not apparent. However, the association of lesions in the globus pallidus with depression and apathy, as well as the diminished reward effects of drugs of abuse, suggest roles in reward and motivation [35].

One of the major points of uncertainty in BD has centered on potential volumetric changes in the hippocampus. In this study, no significant volumetric difference was detected in the hippocampus between BD patients and healthy controls. Two large single-site studies reported a significant hippocampal reduction [36, 37], while other multisite studies reported no significant differences [26-28, 31].

This study did not find any significant change in the mean volume of the thalamus between $\mathrm{BD}$ patients and healthy controls. This is concordant with the results of multiple meta-analyses [26-28, 31], whose findings describe absence of any significant change in the size of the thalamus between BD patients and healthy controls. On the other hand, some studies like Rimol et al. [36] described reduction in the thalamus volume in BD patients.

This study did not find any significant volumetric change in the amygdale between $\mathrm{BD}$ patients and the healthy controls. This is consistent with previous literature-based meta-analysis which could not detect a case-control volumetric difference in the amygdala [37]. However, individual studies in adults with BD had reported either increase or no change in the amygdala volume [38, 39]. In contrast, reduced amygdale volume has been reported in adolescents with BD [40] and has been attributed to developmental abnormalities in this region in adolescence.

In this study, there was no significant difference in the volume of the cortical gray matter of both cerebral hemispheres in BD patients compared to healthy controls. These findings are consistent with those of Yüksel et al. [41] and Rimol et al. [42] who described absence of any volumetric changes in the cortex of both cerebral hemispheres.

In this study, there was no significant change in the volume of the cerebral white matter of both cerebral hemispheres. Our findings are concordant with multiple meta-analysis studies [26-28] which show that the total cerebral white matter volume in BD is preserved without a change.

It should be considered when interpreting the results of neuroantomical studies that abnormalities of structure do not necessarily mean abnormalities of function. In the absence of clinico-anatomical correlations, it is not possible to directly demonstrate that the structure is involved in the expression of BD. Actually, it cannot be determined whether the volumetric abnormalities preceded or, alternatively, developed during the course of disease [43].

To the best of the knowledge of the authors of this study, this work is the first to try to detect correlations 
between structural volumetric changes and different clinical aspects of bipolar I mania, including the age of onset of the disease, the duration of illness, the number of manic attacks, and the YMRS of the patient. No similar studies were found despite extensive research in available published scientific database.

The present study has some potential limitations. One of the limitations is that the global cortical gray matter of both cerebral hemispheres was studied without specifically examining the cortical regions implicated in emotion and cognitive processing and emotion regulation, including the orbitofrontal, anterior cingulate, and anterior temporal cortices. Another limitation is the relatively small sample size of patients and healthy controls, which might affect the sensitivity and specificity of the results; a larger independent sample is needed to confirm the reproducibility of findings.

\section{Conclusions}

VBM could address specific structural findings in the brain in patients with mood disorder bipolar I mania that may contribute to the pathophysiology of the disease. The findings of this study also show that there is significant correlation of these structural changes with different clinical aspects of the disease. These findings encourage similar studies in different neuropsychological disorders.

\section{Abbreviations \\ 3D-T1W-FFE: 3-dimensional T1-weighted fast field echo; BD: Bipolar disorder; CSF: Cerebrospinal fluid; CSPTC: Cortico-striato-pallido-thalamo-cortical; dcm: DICOM format; dem2nii: DICOM to NIfTI format; DICOM: Digital imaging and communication; DSM: Diagnostic and Statistical Manual of mental disorders; FLAIR: Fluid-attenuation inversion recovery; GM: Gray matter; MR: Magnetic resonance; MRI: Magnetic resonance imaging; NIfTI: Neuroimaging Informatics Technology Initiative; nii: NIfTI format; SD: Standard deviation; VBM: Voxel-based morphometry; WM: White matter; YMRS: Young Mania Rating Scale}

\section{Acknowledgements}

The authors of this research thank Dr. Gehan Seifeldein, Assistant Professor of Radiodiagnosis, Faculty of Medicine, Assiut University, Egypt, for helping with the data post-processing and statistical analysis.

\section{Authors' contributions}

HAY is an Assistant Professor of Radiodiagnosis, Faculty of Medicine, Assiut University. This is the corresponding author, who contributed to the hypothesis of the research, shared in the study design and implementation of the methods of the study, scheduled the stages of the study, shared in the processing and analysis of the acquired data. Also, the author is the main contributor in collecting the scientific data and writing down the research, and preparing for publication. The author was responsible for submission of the scientific paper to the journal. YMBS is a Professor of Psychiatry, Faculty of Medicine, Assiut University. This co-author contributed to the hypothesis of the research, shared in its design, performed the clinical evaluation of the subjects, organized, and analyzed the clinical data. Also, this co-author shared in the collection of scientific data and contributed in the writing of the research article. SMA is a Lecturer of Radiodiagnosis, Faculty of Medicine, Assiut University. This co-author contributed to the hypothesis of the research, shared in its design, shared in collection and organization of imaging data, shared in the collection of scientific data, and also contributed in the writing of the research article. SRA is a Specialist in Radiodiagnosis Department, Assiut University Hospitals, Assiut University. This co-author handled the official paperwork of the study with different official authorities and committees, handled the participants of the study, collected the consents, organized the personal data, arranged the scheduling of MR examinations, and handled the subjects during the MRI procedure, collected the imaging data for processing, and prepared the data sheets for statistics. The co-author also shared in the collection of the scientific data. All authors read and approved the final manuscript.

\section{Funding}

The authors state clearly that they did not receive any fund, grant or financial support during the preparation and implementation of this research work from any official, non-official. or commercial organization.

\section{Availability of data and materials}

On behalf of the authors of the paper entitled "Voxel-based morphometry in patients with mood disorder bipolar I mania in comparison to normal controls", me herein, the corresponding author, admit that all the original scientific data and materials of this work are available.

\section{Ethics approval and consent to participate}

The protocol, design, and procedures of this study were approved by the Ethical Committee Board of the Faculty of Medicine of Assiut University, Egypt. The registration ID for this clinical trial is NCT03181698. Informed written consents were obtained from the parents or responsible spouses of the participating patients. Informed consents were also obtained from the healthy control subjects who were included in the study. Confidentiality was maintained throughout the whole study.

\section{Consent for publication}

Dear Editor,

On behalf of the authors of the paper entitled "Voxel-based morphometry in patients with mood disorder bipolar I mania in comparison to normal controls", me herein, the corresponding author, admit that all authors have formally consented for publication of this research work in your respected journal.

All patients included in this research gave written informed consent to publish the data contained within this study. If the patient was less than 16 years old, deceased, or unconscious when consent for publication was requested, written informed consent for the publication of this data was given by their parent or legal guardian.

\section{Competing interests}

The authors declare that they have no competing interests.

\section{Author details}

${ }^{1}$ Radiodiagnosis Department, Faculty of Medicine, Assiut University Hospitals, Assiut University, Assiut University Street, Assiut 71515, Egypt. ${ }^{2}$ Neurology and Psyciatry Department, Faculty of Meicine, Assiut University, Assiut, Egypt.

Received: 7 October 2019 Accepted: 18 December 2019

Published online: 10 January 2020

\section{References}

1. Phillips ML, Kupfer DJ (2013) Bipolar disorder 2 - bipolar disorder diagnosis: challenges and future directions. Lancet 381:1663-1671

2. Solé B et al (2017) Cognitive impairment in bipolar disorder: treatment and prevention strategies. Int J Neuropsychopharmacol 20:670-680

3. Phillips ML, Ladouceur CD, Drevets WC (2008) A neural model of voluntary and automatic emotion regulation: implications for understanding the pathophysiology and neurodevelopment of bipolar disorder. Mol Psychiatry 13:833-857

4. Tohen M (2017) Treatment guidelines in bipolar disorders and the importance of proper clinical trial design. Int J Neuropsychopharmacol 20:95-97

5. Johnson JM, Legesse B, Camprodon JA, Murray E, Price BH (2013) The clinical significance of bilateral basal ganglia calcification presenting with mania and delusions. J Neuropsychiatry Clin Neurosci 25:68-71

6. Johnston JAY et al (2017) Multimodal neuroimaging of frontolimbic structure and function associated with suicide attempts in adolescents and young adults with bipolar disorder. Am J Psychiatry 174:667-675 
7. Ashburner J, Friston KJ (2005) Unified segmentation. Neuroimage 26: 839-851

8. Good CD et al (2001) A voxel-based morphometric study of ageing in 465 normal adult human brains. Neuroimage 14:21-36

9. Manjón JV, Coupé P (2016) Volbrain: an online MRI brain volumetry system. Front Neuroinform 10:30. https://doi.org/10.3389/fninf.2016.00030

10. Strakowski SM (2013) Integration and consolidation. In: The Bipolar Brain. Oxford University Press, pp 253-274. https://doi.org/10.1093/med/ 9780199797608.003.0109

11. Phillips ML, Swartz HA (2014) A critical appraisal of neuroimaging studies of bipolar disorder: toward a new conceptualization of underlying neural circuitry and a road map for future research. Am J Psychiatr 171:829-843

12. Townsend J, Altshuler LL (2012) Emotion processing and regulation in bipolar disorder: a review. Bipolar Disord 14:326-339

13. Yamasaki H, Labar KS, Mccarthy G (2002) Dissociable prefrontal brain systems for attention and emotion. Proc Natl Acad Sci U S A 99:1144711451

14. Chen YC, Thaler D, Nixon PD, Stern CE, Passingham RE (1995) The functions of the medial premotor cortex - II. The timing and selection of learned movements. Exp Brain Res 102:461-473

15. Lane RD et al (1998) Neural correlates of levels of emotional awareness: evidence of an interaction between emotion and attention in the anterior cingulate cortex. J Cogn Neurosci 10:525-535

16. Phan KL, Wager T, Taylor SF, Liberzon I (2002) Functional neuroanatomy of emotion: a meta-analysis of emotion activation studies in PET and fMRI. Neurolmage 16:331-348

17. Zhou Q et al (2017) Trait-related cortical-subcortical dissociation in bipolar disorder: analysis of network degree centrality. J Clin Psychiatry 78:584-591

18. Mayberg HS (2003) Modulating dysfunctional limbic-cortical circuits in depression: towards development of brain-based algorithms for diagnosis and optimised treatment. Br Med Bull 65:193-207

19. Middleton FA, Strick PL (2001) A revised neuroanatomy of frontalsubcortical circuits. In: Lichter DG, Cummings JL, editors. Frontal-Subcortical Circuits in Psychiatric and Neurological Disorders. Guilford Press, New York, pp 44-58

20. Houenou J et al (2012) Neuroimaging biomarkers in bipolar disorder. Front Biosci - Elit 4(E):593-606

21. Strakowski SM et al (2012) The functional neuroanatomy of bipolar disorder: a consensus model. Bipolar Disord 14:313-325

22. Wessa M, Kanske P, Linke J (2014) Bipolar disorder: a neural network perspective on a disorder of emotion and motivation. Restor Neurol Neurosci 32:51-62

23. Sacchet MD, Livermore EE, Iglesias JE, Glover GH, Gotlib IH (2015) Subcortical volumes differentiate major depressive disorder, bipolar disorder, and remitted major depressive disorder. J Psychiatr Res 68:91-98

24. Mamah D, Alpert Kl, Barch DM, Csernansky JG, Wang L (2016) Subcortical neuromorphometry in schizophrenia spectrum and bipolar disorders. Neurolmage Clin 11:276-286

25. Aguilar-Ortiz S et al (2018) Abnormalities in gray matter volume in patients with borderline personality disorder and their relation to lifetime depression: a VBM study. PLoS One 13(2):e0191946. https://doi.org/10.1371/ journal.pone.0191946

26. McDonald C et al (2004) Meta-analysis of magnetic resonance imaging brain morphometry studies in bipolar disorder. Biol Psychiatry 56:411-417

27. Kempton MJ, Geddes JR, Ettinger U, Williams SCR, Grasby PM (2008) Metaanalysis, database, and meta-regression of 98 structural imaging studies in bipolar disorder. Arch Gen Psychiatry 65:1017-1032

28. Arnone D et al (2009) Magnetic resonance imaging studies in bipolar disorder and schizophrenia: meta-analysis. Br J Psychiatry 195:194-201

29. Hibar D, Westlye L, Thompson P, Andreassen O. Enigma Bipolar disorder working group findings from 1,747 cases and 2,615 controls Submission Number: 3376 Authors. https://ww4.aievolution.com/hbm1401/index. $\mathrm{cfm}$ ?do=abs.viewAbs\&subView $=1$ \&abs=3795

30. Van Erp TGM et al (2016) Subcortical brain volume abnormalities in 2028 individuals with schizophrenia and 2540 healthy controls via the ENIGMA consortium. Mol Psychiatry 21:547-553

31. Hallahan B et al (2011) Structural magnetic resonance imaging in bipolar disorder: an international collaborative mega-analysis of individual adult patient data. Biol Psychiatry 69:326-335
32. Edmiston EE et al (2011) Lateral ventricle volume and psychotic features in adolescents and adults with bipolar disorder. Psychiatry Res - Neuroimaging 194:400-402

33. Strakowski SM et al (2002) Ventricular and periventricular structural volumes in first- versus multiple-episode bipolar disorder. Am J Psychiatry 159:1841-1847

34. Bearden CE, Hoffman KM, Cannon TD (2001) The neuropsychology and neuroanatomy of bipolar affective disorder: a critical review. Bipolar Disord 3:106-150

35. Singh A, Levin J, Mehrkens JH, Bötzel K (2011) Alpha frequency modulation in the human basal ganglia is dependent on motor task. Eur J Neurosci 33:960-967

36. Rimol LM et al (2010) Cortical thickness and subcortical volumes in schizophrenia and bipolar disorder. Biol Psychiatry 68:41-50

37. Fears SC et al (2014) Multisystem component phenotypes of bipolar disorder for genetic investigations of extended pedigrees. JAMA Psychiatry 71:375-387

38. Altshuler LL et al (2000) An MRI study of temporal lobe structures in men with bipolar disorder or schizophrenia. Biol Psychiatry 48:147-162

39. Chang K et al (2005) Reduced amygdalar gray matter volume in familial pediatric bipolar disorder. J Am Acad Child Adolesc Psychiatry 44:565-573

40. Pfeifer JC, Welge J, Strakowski SM, Adler C, Delbello MP (2008) Department of psychiatry, M. P. Meta-analysis of amygdala volumes in children and adolescents with bipolar disorder. J Am Acad Child Adolesc Psychiatry 47:1289-1298

41. Yüksel C et al (2012) Gray matter volume in schizophrenia and bipolar disorder with psychotic features. Schizophr Res 138:177-182

42. Rimol LM et al (2012) Cortical volume, surface area, and thickness in schizophrenia and bipolar disorder. Biol Psychiatry 71:552-560

43. Strakowski SM et al (1999) Brain magnetic resonance imaging of structural abnormalities in bipolar disorder. Arch Gen Psychiatry 56:254-260

\section{Publisher's Note}

Springer Nature remains neutral with regard to jurisdictional claims in published maps and institutional affiliations.

\section{Submit your manuscript to a SpringerOpen ${ }^{\circ}$ journal and benefit from:}

- Convenient online submission

- Rigorous peer review

- Open access: articles freely available online

High visibility within the field

- Retaining the copyright to your article

Submit your next manuscript at $>$ springeropen.com 